\title{
Prolapse of posterior leaflet of tricuspid valve presenting as an undulating artifact in right atrium: A rare case report
}

\author{
RK Gokhroo ${ }^{1}$, Kamal Kishor ${ }^{2}$, Bhanwar Ranwa ${ }^{2}$, A. Avinash ${ }^{2}$, Devendra Singh Bisht ${ }^{2}$, Sajal \\ Gupta $^{2}$
}

1. Department of cardiology, Jawaharlal Nehru Medical College Ajmer, Rajasthan, India. 2. Department of cardiology, Jawaharlal Nehru Medical College Ajmer, Rajasthan, India.

Correspondence: Devendra Singh Bisht. Address: Department of cardiology, Jawaharlal Nehru Medical College Ajmer, Rajasthan, India. Email: bisht2416@gmail.com

Received: March 4, 2014

DOI : $10.5430 /$ crim.v1n2p176
Accepted: April 15, 2014

URL: http://dx.doi.org/10.5430/crim.v1n2p176

\section{Abstract}

We present a case of prolapsed posterior leaflet of tricuspid valve presenting as rapidly undulating serpentine mass in mid cavity of the right atrium during ventricle systole mimicking Chiari network. 3D echocardiography unveiled the mystery and confirmed prolapsed posterior leaflet of tricuspid valve. To the best of our knowledge such a large hypermobile artifactual echocardiographic manifestation of prolapsed posterior leaflet of tricuspid valve has not been reported in the literature.

\section{Keywords}

Tricuspid valve prolapse, Artifact, 3D echocardiography

\section{Introduction}

Right atrium structural abnormality is a well known entity in Complete Transposition of Great Arteries (D-TGA) patients ${ }^{[1]}$. Here we are highlighting a case of 2 month old child presenting with D-TGA and a hyper mobile oscillating echo mass in the right atrium (RA). 3D echocardiography confirmed this mass as structurally abnormal posterior leaflet of the tricuspid valve with peculiar movements during different phases of the cardiac cycle mimicking Chiari network.

\section{Case report}

A 2 month old child presented to us with history of intermittent sweating after meals. The relevant physical findings were cyanosis, single S2 and III/VI systolic murmur. 2D echocardiography confirmed the diagnosis of D-TGA, ventricular septal defect and tricuspid regurgitation (TR). Apart from the diagnostic findings of D-TGA on 2D echocardiography, it showed a long thin hyper mobile echo mass in the mid cavity of right atrium which showed oscillating and rotatory movements along a fixed point. Apical four chamber (A4C) and Subcostal views revealed rotatory movements of this mass across the tricuspid valve into the right ventricle (RV), with largest excursions occurring during atrial contraction in late diastole, followed again by rapid motion of this mass into the right atrium at the onset of systole (see Figure 1). 3D echocardiography showed the attachment of the mass to posterior wall with peculiar movements during cardiac cycle, confirming this echo mass as the posterior leaflet of TV (see Figure 2). 

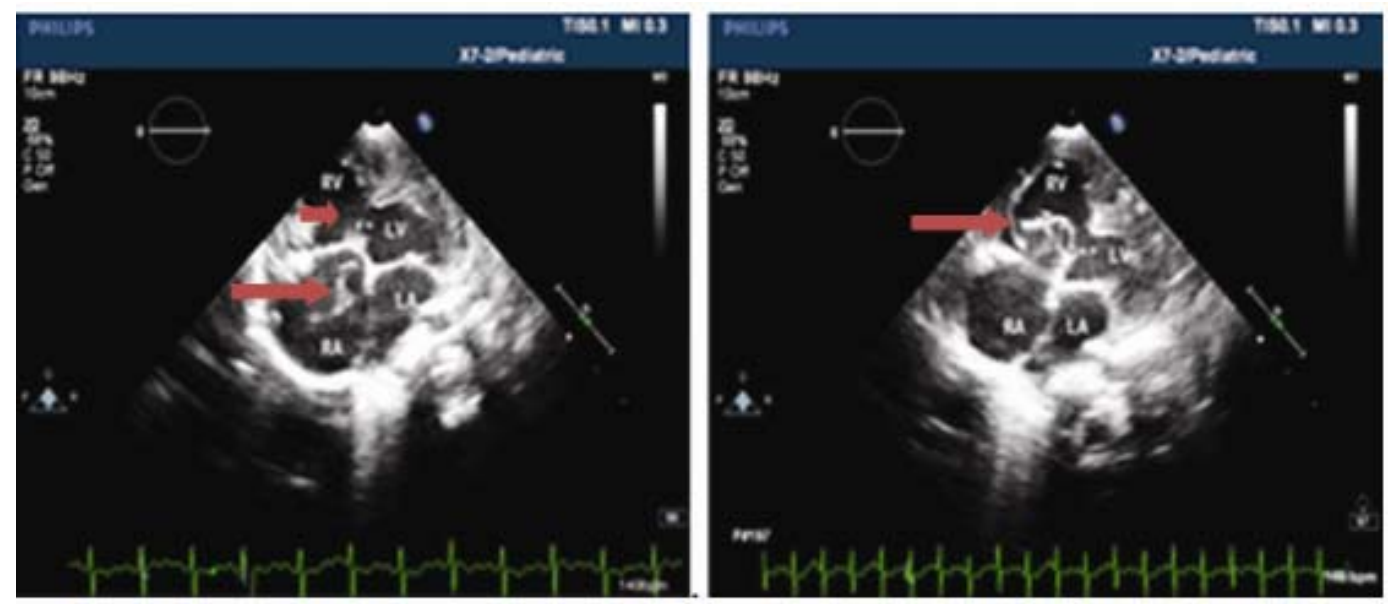

Figure 1. Long thin freely mobile echo mass (large arrow) in RA during systole (left) followed by rapid movement in right ventricle in late diastole. Ventricle septal defect (small arrow) is also seen. RA, Right atrium; RV, Right ventricle; LV, Left ventricle.
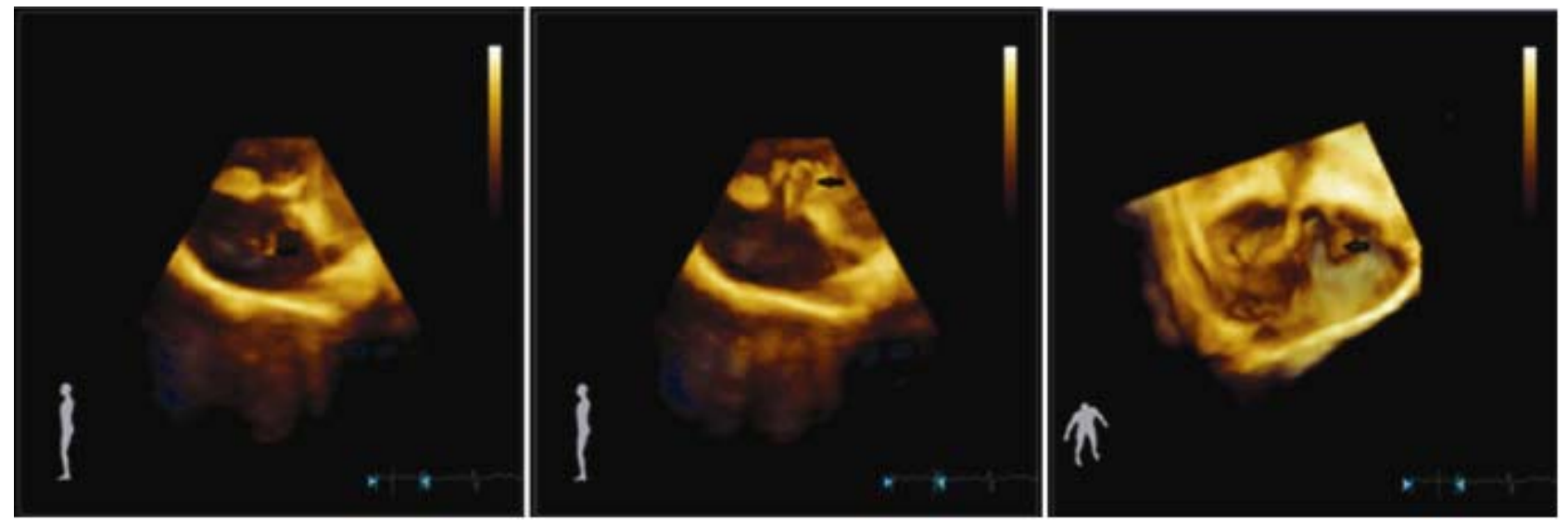

Figure 2. Cropped 3D echocardiography images after acquisition full volume mode using matrix transducer on Philips iE33. Left and middle panel: Cropped images from lateral aspect during systole (left panel) and late diastole (middle panel) confirmed the attachment of oscillating echo mass (black arrow) to TV annular ring on posterior aspect. Right panel: Cropped image has been rotated anteriorly making attachment of echo mass (black arrow) on posterior aspect of tricuspid annulus more appreciable.

\section{Discussion}

Prominent hypermobile mass in the clinical background of congenital cyanotic heart disease with a regurgitant lesion is a source of great concern in light of being nidus for infective endocarditis. Commonest differential diagnosis in the present case is a Chiari network ${ }^{[2,3]}$ (a fenestrated remnant of right valve of sinus venosus resulting from incomplete regression during embryogenesis). Other differentials diagnosis for such a curvilinear, highly mobile, cyclically recurring echo mass include; vegetation on tricuspid valve, ruptured chordae tendineae to the tricuspid apparatus, a small right-atrium thrombus or a pedunculated right-heart tumor. The newer modality of 3D echocardiography is of immense value to answer such clinical puzzle and reach to a conclusive diagnosis. Physiologically it is important because apart from causing TR, the prolapsed leaflet may also be responsible for entrapment of a right-heart catheter, may predispose to thrombus formation and serve as a potential source of pulmonary emboli. Reported anomalies of tricuspid valve in D-TGA patients include; 
severe straddling preventing VSD closure, valve dysplasia requiring anuloplasty or valve replacement and abnormal tricuspid valve chordae attachment to the superior aspect of VSD interfering with Rastelli repair. This patient may have required anuloplasty or valve replacement along with standard surgical repair but unfortunately the patient was lost to follow up ${ }^{[4]}$. We would like to emphasis the unique association of isolated structurally abnormal prolapsing posterior leaflet of TV with D-TGA and the role of 3D echocardiography without which the above diagnostic dilemma would have remained an enigma.

\section{References}

[1] Carole A. Warnes: Transposition of the Great Arteries. Circulation. 2006; 114: 2699-2709. http://dx.doi.org/10.1161/CIRCULATIONAHA.105.592352

[2] Schneider B, Hofmann T, Justen MH, Meinertz T: Chiari’s network: normal anatomic variant or risk factor for arterial embolic events? J Am CollCardiol. 1995 Jul; 26(1): 203-10. http://dx.doi.org/10.1016/0735-1097(95)00144-O

[3] J A Werner, M D Cheitlin, B W Gross, S M Speck and T D Ivey: Echocardiographic appearance of the Chiari network: differentiation from right-heart Circulation. 1981; 63: 1104-1109.

[4] Huhta JC, Edwards WD, Danielson GK, Feldt RH. Abnormalities of the tricuspid valve in complete transposition of the great arteries with ventricular septal defect. J ThoracCardiovasc Surg. 1982 Apr; 83(4): 569-76. 